\title{
In-situ crystallization of GeTelGaSb phase change memory stacked films
}

\author{
A. Velea,,${ }^{1,2, a)}$ C. N. Borca, ${ }^{1}$ G. Socol, ${ }^{3}$ A. C. Galca, ${ }^{2}$ D. Grolimund, ${ }^{1}$ M. Popescu, ${ }^{2}$ \\ and J. A. van Bokhoven ${ }^{1,4}$ \\ ${ }^{1}$ Paul Scherrer Institute, CH-5232 Villigen-PSI, Switzerland \\ ${ }^{2}$ National Institute of Materials Physics, RO-077125 Magurele, Ilfov, Romania \\ ${ }^{3}$ National Institute for Laser, Plasma and Radiation Physics, RO-077125 Magurele, Ilfov, Romania \\ ${ }^{4}$ Institute for Chemistry and Bioengineering, ETH Zurich, CH-8093 Zurich, Switzerland
}

(Received 1 July 2014; accepted 18 November 2014; published online 19 December 2014)

\begin{abstract}
Single and double layer phase change memory structures based on GeTe and GaSb thin films were deposited by pulsed laser deposition (PLD). Their crystallization behavior was studied using in-situ synchrotron techniques. Electrical resistance vs. temperature investigations, using the four points probe method, showed transition temperatures of $138^{\circ} \mathrm{C}$ and $198^{\circ} \mathrm{C}$ for $\mathrm{GeTe}$ and $\mathrm{GaSb}$ single films, respectively. It was found that after GeTe crystallization in the stacked films, Ga atoms from the GaSb layer diffused in the vacancies of the GeTe crystalline structure. Therefore, the crystallization temperature of the Sb-rich GaSb layer is decreased by more than $30^{\circ} \mathrm{C}$. Furthermore, at $210^{\circ} \mathrm{C}$, the antimony excess from GaSb films crystallizes as a secondary phase. At higher annealing temperatures, the crystalline $\mathrm{Sb}$ phase increased on the expense of GaSb crystalline phase which was reduced. Extended X-ray absorption fine structure (EXAFS) measurements at the $\mathrm{Ga}$ and $\mathrm{Ge} \mathrm{K}$-edges revealed changes in their local atomic environments as a function of the annealing temperature. Simulations unveil a tetrahedral configuration in the amorphous state and octahedral configuration in the crystalline state for Ge atoms, while $\mathrm{Ga}$ is four-fold coordinated in both as-deposited and annealed samples. (c) 2014 AIP Publishing LLC.
\end{abstract}

[http://dx.doi.org/10.1063/1.4904741]

\section{INTRODUCTION}

Due to modern technologies, the needs for storage capacity have increased exponentially. The most used memories today are nonvolatile memories. Among these, the most popular nonvolatile memories are Flash memories which are based on charge storage. Because charge storage memories are facing miniaturization problems, ${ }^{1}$ some possible replacements are increasingly investigated. While magnetic and ferroelectric random access memories have problems with scalability, ${ }^{2}$ the nonvolatile memories based on resistance change rather than charge storage seem to take the lead. In this class of resistive memories, we find phase change memories (PCMs). PCMs store data in a glass containing chalcogens ( $\mathrm{S}, \mathrm{Se}$, or $\mathrm{Te}$ ) or pnictogens (such as $\mathrm{Sb}$ ) and use low voltage (or ultra-fast laser pulses) to switch subdomains of the material between two different states. ${ }^{3}$ In one state, the atoms of the glass are arranged in a disordered amorphous lattice (so called "off" state or digital "0"), while in the other state they have a crystalline distribution ("on" state or digital " 1 "). The transition from amorphous to crystalline is made using a pulse of intermediate power which locally heats the material above the glass transition temperature $\left(T_{g}\right)$ and the material switches into the crystalline state. For phase change materials, $\mathrm{T}_{\mathrm{g}}$ is only a few degrees below the crystallization temperature $\left(\mathrm{T}_{\mathrm{x}}\right) .{ }^{4}$ This transition is accompanied by large variations in optical reflectivity (the refractive index is up to $30 \%$ higher in the crystalline phase) and electrical conductivity (the electrical resistance is several

${ }^{\text {a)} E l e c t r o n i c ~ m a i l: ~ a l i n . v e l e a @ p s i . c h . ~}$ orders of magnitude higher in the amorphous phase). Also, a decrease in sample thickness is produced (usually of a few percent, which will increase the material density). To switch a PCM memory cell back into the "off" state, a short intense pulse is used. The material is heated above the melting temperature and by rapid cooling $\left(10^{10} \mathrm{~K} / \mathrm{s}\right)$ it quenches into a disordered state. Usually, the crystallization is a slow process but due to the fact that phase change materials are poor glass formers ${ }^{5}$ they can be switched between these two states in a few nanoseconds. Several techniques such as ab initio molecular dynamics, ${ }^{6}$ Monte Carlo, ${ }^{7}$ and cellular automata ${ }^{8}$ have been used to model this transition.

Regarding the crystallization processes, there are two classes of phase change materials: nucleation dominated and growth dominated materials. ${ }^{5}$ In the first case, crystalline nuclei grow in the amorphous region while in the second case, the crystalline regions grow from the margins to the volume of the material. For phase change materials crystallization usually occurs at the interface. The nucleation dominated materials are characterized by long incubation times, while the nucleation sites are rare. The incubation time can be reduced by applying a weak electric field $(0.3 \mathrm{~V})$ to produce a pre-ordering of the amorphous clusters via Joule heating, leading to less than $1 \mathrm{~ns}$ switching times. ${ }^{9}$ Growth dominated materials are characterized by a strong dependence of the crystallization time on the size of the amorphous region, nucleation is very sparse and a supercritical nucleus size is needed in order for the crystallization to occur. After the formation of a crystalline nucleus, the crystallization proceeds through the rapid movement of the amorphous/crystalline boundary. 
In the last years, attention was turned to binary PCM like $\mathrm{GeTe}^{10}$ and $\mathrm{GaSb}^{11}$ as promising candidates for technological applications since they combine high speed with good thermal stability. GeTe is a growth dominated material $^{12}$ with a crystallization time of a few nanoseconds ${ }^{13}$ achieved using Nd:YAG laser pulses and the and measured with optical microscopy and TEM near the crystallization temperature. It is considered one of the best materials for phase change memories. ${ }^{14} \mathrm{GaSb}$ is a nucleation-dominated material, with a high thermal stability due to a very stable amorphous phase $\mathrm{e}^{15}$ and a fast crystallization time $(<15 \mathrm{~ns}$ obtained when heated to temperatures close to their melting point). ${ }^{16} \mathrm{GaSb}$ shows negative optical contrast (i.e., the refractive index is smaller in the crystalline phase than in the amorphous one, which is not a typical aspect of phase change materials) upon crystallization, but this do not affect electronic memories. $\mathrm{Sb}$ is often proposed for replacing $\mathrm{Te}$ and has the potential to offer a fast phase transition due to minimal changes in the atomic rearrangement from the amorphous to the crystalline phase. ${ }^{17}$

What is exciting about PCMs is that multiple states can be recorded in a single PCM memory cell. ${ }^{18,19}$ There are several approaches to achieve this goal. One is to store multiple states in a single film phase change material by controlling the crystalline to amorphous ratio in the thin film. For example, $\mathrm{AlSb}^{20}$ exhibits three distinct resistance levels due to the co-existence of crystalline Sb-rich regions in an Al-rich amorphous matrix. Unfortunately, this approach suffers from resistance drift $^{21}$ over time and cannot ensure long time stability of the memory cell. Another approach is to stack different films of chalcogenide materials. ${ }^{22,23}$ Each state corresponds to a different value of the cell resistance and to a different combination of the layers structure. Because this second approach is more robust in terms of stability we chose to use it in our experiments.

An important drawback of phase change memory materials is void formation ${ }^{24}$ during cycling due to the large difference in density between the two phases. This phenomenon leads to elemental segregation which is a common failure mechanism observed in PCM cells using GST $\left(\mathrm{Ge}_{2} \mathrm{Sb}_{2} \mathrm{Te}_{5}\right)$ as a phase change material. The defects induced in the material by these effects can be beneficial at the beginning, leading to an increase in the switching speed ${ }^{25}$ but also to cell failure when a certain amount of the material is affected. Antimony-rich areas are usually formed near the electrodes ${ }^{26}$ causing a reduced crystallization temperature and stability. ${ }^{27}$

In order to better understand the crystallization process in stacked structures, we need to know the structure of the materials in the amorphous and crystalline states. This knowledge is critical in revealing the switching mechanism and is useful for improving materials properties. The amorphous state is not stable and is not unique. On the other hand, one would believe that resolving the crystalline structure of phase change materials is a straight forward process with all the modern techniques and should be much easier than for the amorphous phase. Due to the fact that phase change materials have crystallization speed of a few nanoseconds, their crystalline state consists of small crystallites (tens of nanometers) without any preferred orientation. That is why the crystalline phases are usually metastable and they have a lot of similarities with the amorphous phases from whom they originate. This kind of polycrystalline domains lead to low intensity broad diffraction peaks. An idea to solve this problem of structure determination was to investigate single crystals ${ }^{28}$ and resolve their structure but then comes the question of how much resemblance is between single crystals grown close to thermal equilibrium and polycrystalline materials obtained on timescales of nanoseconds and fast cooling rates. Another solution for structure determination, which we have adopted here, is to simultaneously measure by two complementary techniques (Extended X-ray Absorption Fine Structure-EXAFS and X-ray Diffraction-XRD) the short and long-range order during the phase transition. Combined synchrotron techniques like EXAFS and XRD are great tools for the in-situ/ex-situ determination of the local structure around the absorbing atom such as the number of nearest neighbors, their chemical nature, the interatomic distances and the disorder in both the amorphous and the crystalline states. In this paper, we report a comparative study regarding the crystallization behavior of single and stacked films of $\mathrm{GeTe}$ and $\mathrm{GaSb}$.

\section{MATERIALS AND METHODS}

Pulsed laser deposition (PLD) was proved to be a successful method for preparing chalcogenide films with similar stoichiometry as the targets, ${ }^{29}$ on large uniform areas. ${ }^{30}$ It is used for obtaining a broad range of amorphous thin films applicable in optoelectronics, ${ }^{31}$ chemical sensors, ${ }^{32}$ optical waveguides, ${ }^{33}$ and phase change materials. ${ }^{34}$ We believe that PLD is a scalable, versatile, and cost effective method which can be used to grow high quality phase change samples in a very short time. The deposition method should always be taken into account in the case of phase change materials due to the influence on the microstructure and the crystallization temperature, which control the optical and electrical properties of the samples. In some cases, the crystallization temperature of thin films with the same composition of the initial target present differences larger than $200^{\circ} \mathrm{C}$ for various deposition methods and no explanation has been found yet. ${ }^{35}$ The deposition was carried out using a $\mathrm{KrF}^{*}$ laser source, model COMPexPro 205, Lambda Physics-Coherent $\left(\lambda=248 \mathrm{~nm}, \tau_{\mathrm{FWHM}}=25 \mathrm{~ns}\right)$. The targets were irradiated using a $10 \mathrm{~Hz}$ repetition rate and a laser fluence of $1.5 \mathrm{~J} / \mathrm{cm}^{2}$. The depositions were carried out at room temperature and the pressure inside the deposition chamber was $5 \times 10^{-4} \mathrm{~Pa}$. During laser irradiation, the substrates were kept at room temperature. The target-substrate separation distance was $4 \mathrm{~cm}$ for GeTe films and $8 \mathrm{~cm}$ for GaSb films in order to avoid as much as possible the formation of droplets, taking into account that $\mathrm{GaSb}$ is a softer material than GeTe. Due to the difference in the target-substrate separation distance and hardness of the materials, different numbers of laser pulses were used in order to obtain the desired film thicknesses (7500 and 20000 pulses for GeTe and GaSb, respectively).

PLD targets were prepared using high purity powders (5N GeTe and and 4N GaSb), from Alpha Aesar, which were 
loaded in vacuum evacuated quartz ampoules and melted for $2 \mathrm{~h}$ at $800^{\circ} \mathrm{C}$ in a tubular rocking furnace. Finally, the ampoules were slowly cooled until room temperature in air. Thus, ingots of $10 \mathrm{~mm}$ in diameter and $20 \mathrm{~mm}$ in length were obtained and then cut as discs of $2 \mathrm{~mm}$ thickness. In order to provide a good adhesion for the chalcogenide films but also to be transparent as much as possible to X-rays, $200 \mu \mathrm{m}$ thick glass substrates were used. Film thickness and roughness were measured using a non-contact Veeco Dektak 150 profilometer. We obtained films with thicknesses ranging between 200 and $500 \mathrm{~nm}$ and a roughness between 20 and $50 \mathrm{~nm}$. Thickness is another factor which influences the crystallization temperature of phase change materials. ${ }^{36}$ It becomes very important for films with thicknesses below $10 \mathrm{~nm}$, leading to an increase of more than $200^{\circ} \mathrm{C}$ of the crystallization temperature. For thicknesses above $10 \mathrm{~nm}$, the crystallization temperature is more or less the same as compared to the bulk samples. The average composition of the films was measured by means of an X-ray Fluorescence table top system from EDAX, using a beam size of $2 \mathrm{~mm}^{2}$ and an acquisition time of $200 \mathrm{~s}$. The radiation was produced by an Rh cathode using an accelerating voltage of $35 \mathrm{kV}$ and a current of $200 \mathrm{~mA}$. The sample composition is very important for the properties of thin phase change memory films. For example, the crystallization time is several orders of magnitude different for small changes in the material composition $^{37}$ and is very different for as-deposited as opposed to melt quenched samples. ${ }^{38}$ The films obtained in this study were uniform in thickness and composition on an area of $2.5 \mathrm{~cm}^{2}$.

Electrical resistance versus temperature measurements were performed in a custom-made four-point measurement setup using Pt sputtered contacts on film surfaces. The measurements were conducted in Ar atmosphere using continuous heating at a rate of $4{ }^{\circ} \mathrm{C} / \mathrm{min}$. The heating rate is another parameter that influences the crystallization temperature. Thus, an increase in the crystallization temperature of more than $20^{\circ} \mathrm{C}$, when the heating rate was increased from 0.5 to $5.3^{\circ} \mathrm{C} / \mathrm{min}$, was observed. ${ }^{39} \mathrm{XRD}$ and EXAFS measurements were performed at the microXAS beamline ${ }^{40}$ from the Swiss Light Source. In-situ X-ray diffraction was carried out using a hot air blower with a heating rate of $10^{\circ} \mathrm{C} / \mathrm{min}$. Additional X-ray diffraction measurements were performed on samples annealed ex-situ, in Ar atmosphere, at temperatures below and above the transition temperatures of the single layers. The heating rate in this case was $4{ }^{\circ} \mathrm{C} / \mathrm{min}$ and a $2 \mathrm{~h}$ plateau. In order to detect most of the diffraction peaks, XRD spectra were acquired using high energy radiation (17 keV, $\lambda=0.729 \AA$ ), from a monochromator with Si (111) crystals and a small area single photon counting bidimensional detector (100k Pilatus, DECTRIS Ltd.) situated at $5 \mathrm{~cm}$ from the sample. The diffraction patterns were collected in transmission geometry and the observed $2 \theta$ range was between 10 and $45^{\circ}$.

For the computation of crystallite size and microstrain, we used Williamson-Hall analysis. ${ }^{41}$ The peak broadening $(\beta)$ of a reflection located at a $2 \theta$ angle is given by the following formula:

$$
\beta=\frac{K \lambda}{D \cos (\theta)}+\varepsilon \tan (\theta),
$$

where $\mathrm{K}$ is Scherrer's constant, $\lambda$ the wavelength, $\theta$ the angle and $\varepsilon$ is related to the microstrain and D is the crystallite size. By plotting $\beta \cos (\theta)$ as a function of $4 \sin (\theta)$, a straight line is obtained. The microstrain occurring between crystallites is inferred from the slope of this line, while the average crystallites size is determined from the intercept. Gaussian convolution of the experimental peak profiles and a value of 0.9 for the Scherrer constant were used. EXAFS spectra were collected in fluorescence mode on the ex-situ annealed samples using a single element Si drift diode detector (from Ketek, $160 \mathrm{eV}$ resolution). Investigations were performed at the $\mathrm{Ga}(10.36 \mathrm{keV})$ and $\mathrm{Ge}(11.1 \mathrm{keV}) \mathrm{K}$ edges. In both cases, the beam size was $300 \times 300 \mu \mathrm{m}^{2}$. The XRD data were compiled and analyzed using XRDUA ${ }^{42}$ and Match $^{43}$ software packages, while the EXAFS data were analyzed using Demeter. ${ }^{44}$ The simulations were performed using Artemis.

\section{RESULTS AND DISCUSSION}

Single films of GeTe and GaSb with thicknesses of $500 \mathrm{~nm}$ and $200 \mathrm{~nm}$, respectively, as well as double layers of GeTelGaSb $(500 \mathrm{~nm} \backslash 200 \mathrm{~nm})$ were produced by PLD. Roughness measurements showed that GeTe films were smooth with RMS values less than $20 \mathrm{~nm}$, while the GaSb films were rougher with RMS values around $50 \mathrm{~nm}$, mainly due to PLD droplets. The large area films we prepared together with the $300 \times 300 \mu \mathrm{m}^{2} \mathrm{X}$-ray beam size, gave us the possibility to target the zones with the lowest concentration of droplets for XRD and EXAFS measurements. The composition of the single and stacked films measured by XRF with an error of $\pm 2 \%$ is summarized in Table I. The samples are slightly non-stoichiometric with $\mathrm{Ge}$ and $\mathrm{Sb}$, respectively, in excess.

\section{A. GeTe}

It is known that at temperatures below $430{ }^{\circ} \mathrm{C}$, GeTe has a distorted rock-salt structure. ${ }^{45} \mathrm{Ge}$ and Te atoms occupy the cation lattice and the anion lattice positions, respectively. Due to the fact that these two lattices are shifted, the lattice angle decreases and the resulted structure is rhombohedral $(\mathrm{a}=8.342 \AA, \mathrm{c}=10.668 \AA), \mathrm{R} 3 \mathrm{~m}$ space group. Above $430^{\circ} \mathrm{C}$, a transition from the rhombohedral metastable crystalline structure to a rock-salt cubic structure occurs. ${ }^{46}$ This additional transition (which was not achieved in the current experiments because lower annealing temperatures were used) is also accompanied by a drop in resistance (which is usually smaller than the first one) and very often by

TABLE I. Sample composition determined by XRF.

\begin{tabular}{lcccc}
\hline \hline Sample & $G e($ at. \%) & Te (at. \%) & Ga (at. \%) & $S b($ at. \%) \\
\hline GeTe & 58 & 42 & $\ldots$ & $\ldots$ \\
GaSb & $\ldots$ & $\ldots$ & 46 & 54 \\
GeTe\GaSb & 58 & 42 & 46 & 54 \\
\hline \hline
\end{tabular}


elemental phase segregation. The mechanism of the structural arrangement during crystallization and amorphization is still unclear. In most amorphous solids, their short range order resembles the structure of their corresponding crystals. $\mathrm{GeTe}$ is an exception from this rule and its structure in the amorphous and crystalline states has not been entirely revealed. Another counterintuitive fact is that the $\mathrm{Ge}-\mathrm{Te}$ bond is longer (i.e., weaker) in the crystalline state than in the amorphous structure. ${ }^{47}$ In the amorphous phase both homopolar $\mathrm{Ge}-\mathrm{Ge}$ and heteropolar $\mathrm{Ge}-\mathrm{Te}$ bonds exist in the first coordination shells while after crystallization only $\mathrm{Ge}-\mathrm{Te}$ bonds exist and they increase significantly in length. It was shown that in the amorphous state there is a random covalent network with Ge four fold coordinated and Te two fold coordinated, ${ }^{48,49}$ while others suggest that $\mathrm{Ge}$ and $\mathrm{Te}$ are both threefold coordinated. ${ }^{50,51}$ Some other authors do not agree with this theory and consider that the structure is a mix of $4(\mathrm{Ge}): 2(\mathrm{Te})$ and $3(\mathrm{Ge}): 3(\mathrm{Te})$ coordinated structural units. ${ }^{52}$

For a precise determination of the crystallization temperatures in single films, we performed resistance versus temperature (R-T) measurements. Fig. 1 shows the results of the R-T measurements on the GeTe single films. At temperatures below, the crystallization threshold, the films were highly resistive due to their amorphous (insulating) phase and the resistivity decreased gradually until exceeding the crystallization/transition temperature. The conductivity in amorphous materials can be modeled by a thermally activated hopping process, ${ }^{53}$ until a rapid drop in resistance is observed at the transition to the crystalline (conductive) state. The resistivity in the crystalline state does not depend on the annealing temperature because $\mathrm{GeTe}$ is a degenerate semiconductor. ${ }^{54}$ In the case of our GeTe films, the drop in resistance is of 5 orders of magnitude and occurs at a transition temperature of $138^{\circ} \mathrm{C}$. A similar temperature was also reported by Kolobov et $a l .{ }^{47}$ for $5 \mu \mathrm{m}$ thick films obtained by magnetron sputtering onto Kapton substrate. The crystallization temperature was

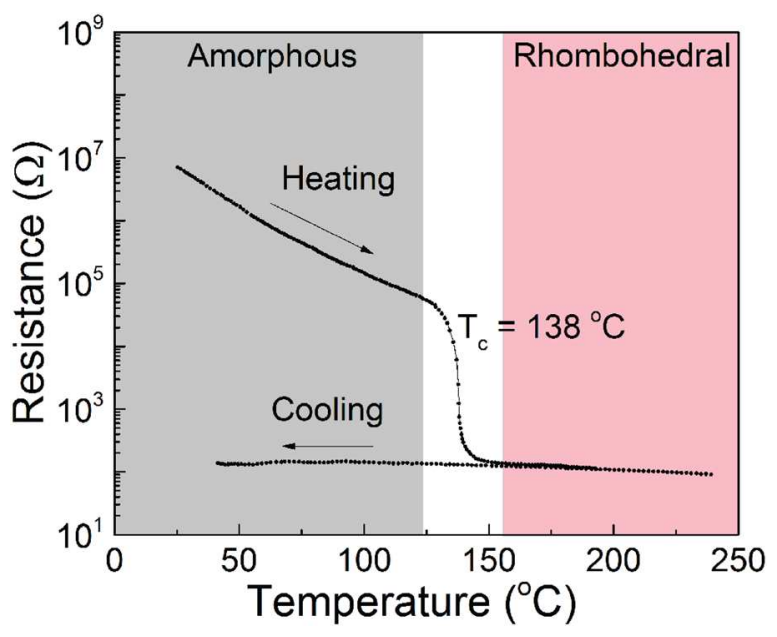

FIG. 1. Typical resistance-temperature measurements on GeTe thin films measured using the four points probe method. The films were measured in Ar atmosphere using continuous heating at $4{ }^{\circ} \mathrm{C} / \mathrm{min}$. Crystallization is indicated by a sudden drop in resistance at $138^{\circ} \mathrm{C}$. determined from the peak in the first order derivative of the resistivity as function of temperature. Upon cooling, the sample remained in the low resistivity state because in our experiments the melting temperature of the material has not been reached and the transition back into the amorphous state was not possible.

Fig. 2 presents the XRD diffractograms recorded on the same GeTe thin films used for temperature-resistance measurements, before and after annealing. GeTe thin films were amorphous and they have crystallized in a rhombohedral structure after annealing. The crystallization is a one-step process characterized by the appearance of X-ray diffraction peaks at one temperature correlated with the sharp drop in resistance at the same temperature. The wide peak centered at $2 \theta=10.7^{\circ}$ is due to scattering from the glass substrate. The intensity of the diffraction peaks was normalized to 1 using GeTe (202) crystalline peak intensity for both as-deposited and annealed samples spectra. The appearance of Ge (111) and $\mathrm{Te}(012)$ peaks at high annealing temperature $\left(250^{\circ} \mathrm{C}\right)$ implies the presence of precipitation without any significant variation of electrical resistance. Ge precipitation was also observed by Raoux et al. ${ }^{55}$ in the case of co-sputtered GeTe samples annealed above $200^{\circ} \mathrm{C}$. The Ge and Te precipitation is due to non-stoichiometric samples and long annealing times. Crystallite size and microstrain were computed using the Williamson-Hall analysis (detailed in Sec. II). For a better estimation only non-overlapping peaks were used. As one can see from the linear regression in Fig. 3, the slope gives a microstrain of $3.55 \times 10^{-3}$ for rhombohedral GeTe and the intercept an average crystallites size of $15 \pm 3 \mathrm{~nm}$.

\section{B. GaSb}

$\mathrm{GaSb}$ has a face-centered cubic structure, ${ }^{56} \mathrm{~F}-43 \mathrm{~m}$ space group, with a lattice constant $\mathrm{a}=6.095$ and an amorphous structure very similar with the crystalline state structure. In the amorphous state, $\mathrm{Ga}$ is surrounded by almost four (3.7) Sb atoms in the first shell, while in the crystalline state $\mathrm{Ga}$ has exactly $4 \mathrm{Sb}$ neighbors. ${ }^{56}$ This small difference

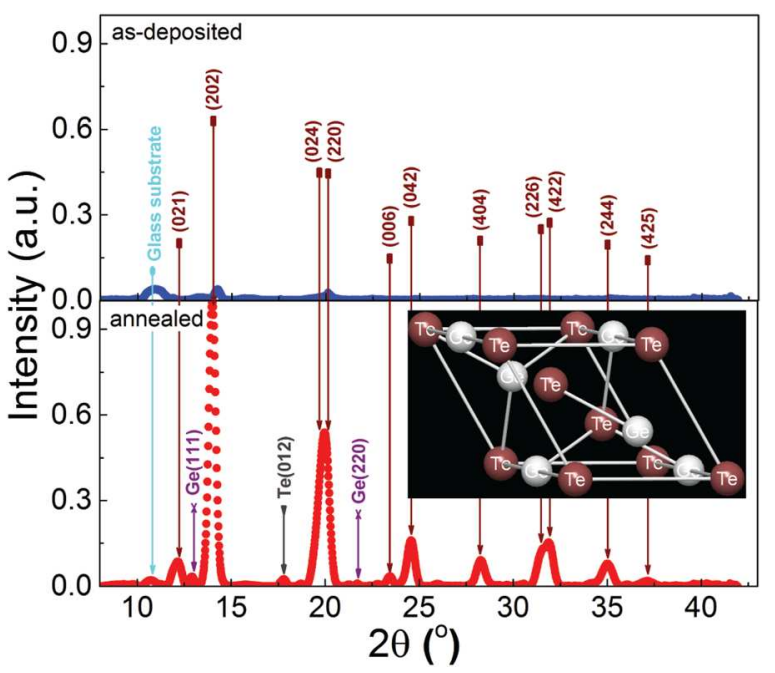

FIG. 2. Typical XRD spectra of GeTe films, before and after annealing at $250^{\circ} \mathrm{C}$. GeTe crystallizes into a rhombohedral structure (inset). Ge and Te phase segregation is observed in the annealed sample. 


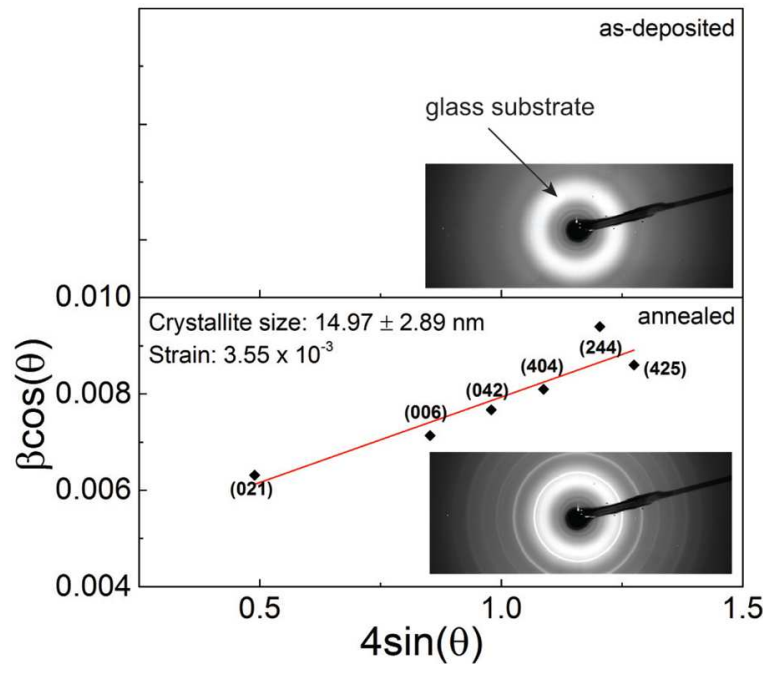

FIG. 3. Williamson-Hall analysis on GeTe films. The crystallites size is approximatively $15 \mathrm{~nm}$. Debye-Scherer rings of as-deposited and annealed samples are shown in the inset.

is due to the presence of wrong $\mathrm{Ga}-\mathrm{Ga}$ bonds in the amorphous phase.

For the gallium antimony sample the transition temperature is at $198^{\circ} \mathrm{C}$ as shown in Fig. 4 and the electrical contrast is of 4 orders of magnitude. Upon cooling the resistance remains lower than in the amorphous phase. The electrical contrast in our GaSb films, which have slightly nonstoichiometric composition, is much stronger than the contrast in stoichiometric $\mathrm{GaSb}$ which was found to be of only one order of magnitude. ${ }^{57}$ The electrical contrast can be increased by annealing at temperatures much higher than the crystallization temperature. ${ }^{23}$ However, this is not a viable solution because high annealing temperatures induce an irreversible segregation process. Gallium antimony is partially crystalline in the as-deposited state and it fully crystallizes after the transition temperature as seen in Fig. 5. All peaks from the XRD diffraction can be identified as cubic GaSb phase. The XRD results are in agreement with the resistivity vs. temperature data. The partial crystallinity of GaSb films, which is mainly due to PLD droplets (SEM measurements showed droplets of

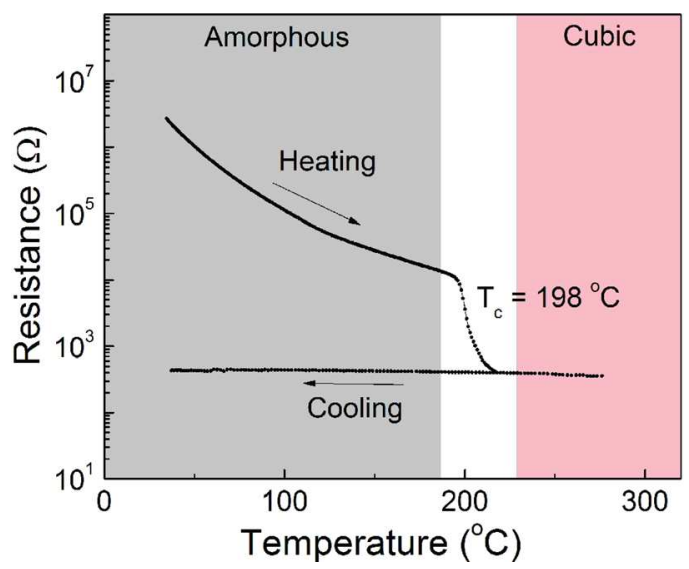

FIG. 4. Electrical resistance-temperature measurements on GaSb thin films measured using the four points probe method. The films were measured in Ar atmosphere using continuous heating at $4{ }^{\circ} \mathrm{C} / \mathrm{min}$. Crystallization is indicated by a sudden drop in resistance at $198^{\circ} \mathrm{C}$.

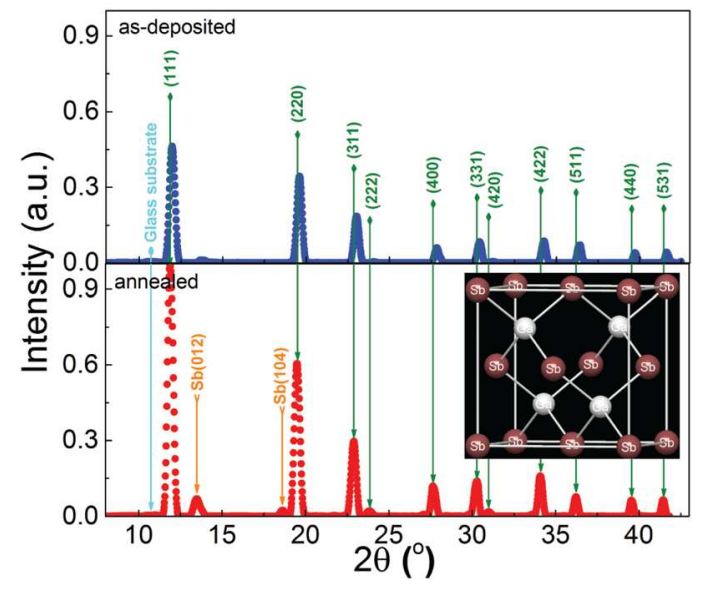

FIG. 5. XRD spectra of GaSb films, before and after annealing. GaSb crystallizes into a cubic structure (inset). Sb crystallization was observed in the annealed samples.

$35-50 \mathrm{~nm}$, data not shown), does not affect the switching effect. The fact that the crystallization temperature of our single layer GaSb films is so close to the value found in the literature $\left(203^{\circ} \mathrm{C}\right)^{11}$ for films prepared using other techniques such as sputter deposition suggests that the influence of droplets is not important in our experiments. In the diffraction pattern, some small amount of metallic phase of $\mathrm{Sb}$ can be detected. The $\mathrm{Sb}$ (012) rhombohedral peak is present in the as-deposited films and increases at high annealing temperatures, where the second peak of the rhombohedral phase appears due to $\mathrm{Sb}$ excess crystallization. Raoux et al. ${ }^{11}$ reported crystalline rhombohedral $\mathrm{Sb}$ in samples deposited at room temperature and observed $\mathrm{Sb}$ segregation at annealing temperatures as high as $350{ }^{\circ} \mathrm{C}$. It was suggested ${ }^{11}$ that materials with an excess of $\mathrm{Sb}$ could be of great interest for multistate phase change materials due to their stepwise crystallization, the first step being the crystallization of $\mathrm{GaSb}$ at lower temperatures and the second step the crystallization of $\mathrm{Sb}$ at higher annealing temperatures. This second drop in resistance was not achieved in our experiments due to lower annealing temperatures. Sb precipitation was also found in GeSb films at high annealing temperatures. ${ }^{58}$ A slight displacement of the diffraction peaks is evidenced in the annealed samples which could be attributed to density change after phase transition. The intensity of the diffraction peaks was normalized to 1 using GaSb (111) crystalline peak intensity for both as-deposited and annealed samples.

The negative slope in the Williamson-Hall plot (Fig. 6) indicates that the microstrain is minimal in the material. ${ }^{59}$ Usually, the full width half maximum (FWHM) increases with $\sin (\theta)$ because the broadening due to microstrain must increase. In the case of GaSb single films FWHM decreases showing that microstrain is not a source of broadening, therefore it is very low. The intercept gives an average crystallites size of $8.5 \mathrm{~nm} \pm 1.5 \mathrm{~nm}$. This estimation of crystallite size indicates a continuous nucleation process in the case of $\mathrm{GaSb}$ as reported also in the literature. An estimation of the lattice constant from the XRD spectra is $6.065 \pm 0.002 \AA$ before annealing and $6.100 \pm 0.002 \AA$ after annealing, which is very close to the published value. ${ }^{56}$ 


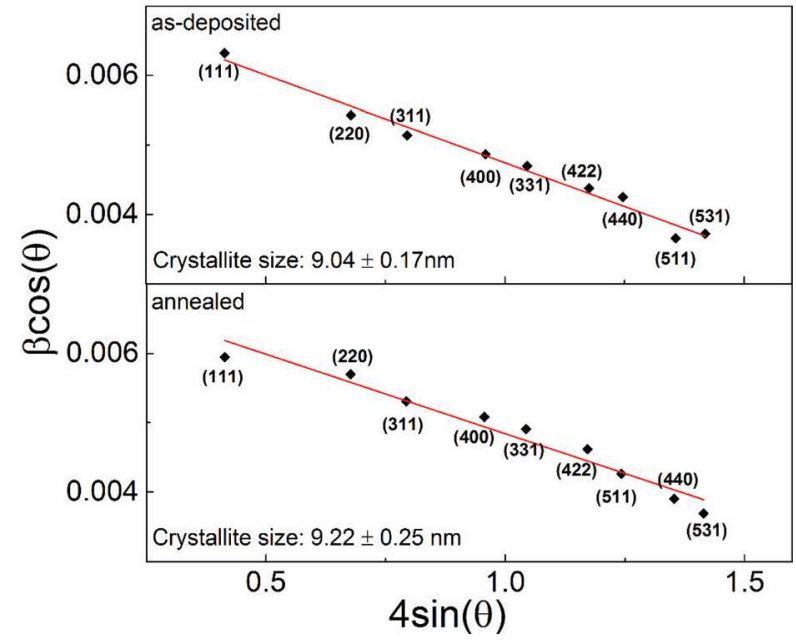

FIG. 6. Williamson-Hall analysis on GaSb thin films. The average crystallites size is approximatively $9 \mathrm{~nm}$ and does not change from the as-deposited to the annealed samples.

\section{GeTe/GaSb}

In order to investigate the possibility of controlling the crystallization of the individual layers, we performed in-situ X-ray diffraction vs. temperature measurements on GeTelGaSb stacked structures. Fig. 7 shows that $\mathrm{GaSb}$ is partially crystalline at room temperature which was also the case for single films. Further increase in temperature determines the appearance of GeTe rhombohedral peaks after $155^{\circ} \mathrm{C}$. Crystallization temperature was estimated by integrating the area of GaSb (111) and GeTe (202) diffraction peaks as a function of temperature and computing the first order derivative maximum. The increase in peak area, due to peak broadening caused by the reduction of grain size or film thickness, was not considered. The crystallization temperatures derived from XRD are usually $10-20{ }^{\circ} \mathrm{C}$ higher compared to electrical vs. resistance measurements. The reason for this is that XRD peaks require a substantial fraction of the material to be in long range ordered crystalline state, while the drop in resistivity requires only the existence of a

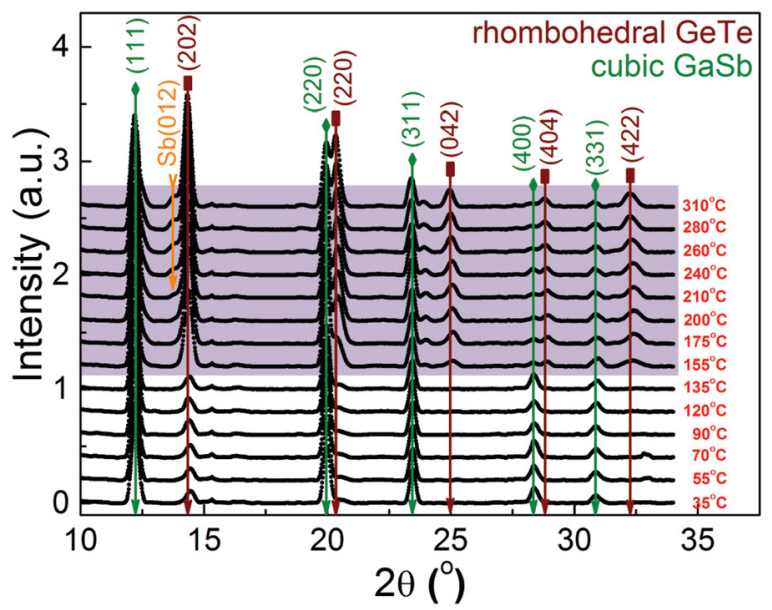

FIG. 7. XRD spectra of GeTelGaSb stacked films during in-situ annealing between $35^{\circ} \mathrm{C}$ and $310^{\circ} \mathrm{C}$. GeTe crystallization occurs at $149^{\circ} \mathrm{C}$ and $\mathrm{Sb}$ crystallizes at $210^{\circ} \mathrm{C}$. The crystallization of GeTe film is highlighted. conduction filament between the electrodes which does not depend on ordered paths.

Fig. 8 denotes a crystallization temperature of $150{ }^{\circ} \mathrm{C}$ for GeTe. After GeTe crystallization, we consider that some of the $\mathrm{Ga}$ atoms (which are the smallest atoms in the structure) diffused in the vacancies of the GeTe crystalline structure (the GeTe crystalline structure contains up to $10 \%$ vacancies). Diffusion produced an increased concentration of $\mathrm{Sb}$ in the GaSb film which will lead to a reduction of the crystallization temperature as shown by Raoux et al. ${ }^{60}$ The crystallization temperature is composition dependent, decreasing with the increase in Sb concentration. Thus, the crystallization temperature of the Sb-rich GaSb layer is decreased to $168^{\circ} \mathrm{C}$. On the other hand, the interface might play a role in the crystallization of GaSb. GeTe droplets and the mismatch between lattice constants of the two films will lead to a stressed interface. This stress will increase after GeTe crystallization and could determine the formation of crystallization nuclei in the GaSb film. These nucleation sites might trigger the crystallization in the GaSb film. Furthermore, $\mathrm{Sb}$, which is known to exhibit an explosive crystallization, ${ }^{61}$ begins to crystallize at $210^{\circ} \mathrm{C}$ as shown by the arrow in Fig. 8. Sb (012) rhombohedral peak starts to be visible at this temperature and then increases with annealing. Further crystallization of antimony produces a drop in GaSb (111) peak intensity (Fig. 8), meaning that after Sb excess crystallizes then the crystalline $\mathrm{Sb}$ phase grows on the expense of GaSb phase which starts to separate. GaSb (400) peak intensity decrease could also be related to antimony crystallization. A similar result was found by Kaiser et al. ${ }^{62}$ They studied the crystallization of bulk Sb-rich alloys by transmission electron microscopy and showed that these alloys are not stable and they separate in $\mathrm{GaSb}$ and $\mathrm{Sb}$. A analogous process was found in GeSb alloys. Del Pozo et $a l .{ }^{63}$ suggested a metallic induced crystallization for $\mathrm{Ge}$ precipitation at temperatures above $\mathrm{Sb}$ crystallization in $\mathrm{GeSb}$ alloys. The process begins with the crystallization of $\mathrm{Sb}$ and its diffusion into the GeSb matrix leading to $\mathrm{Ge}-\mathrm{Sb}$ bond breaking and Ge crystallization.

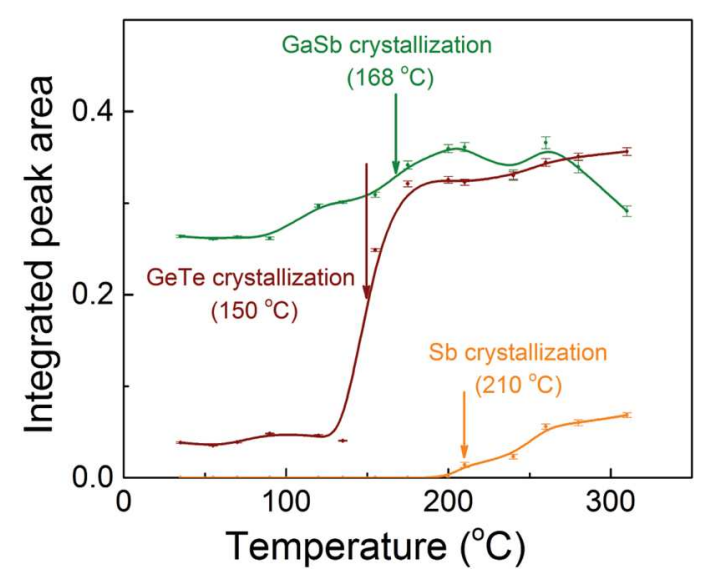

FIG. 8. Normalized integrated areas of GaSb (111) (green), GeTe (202) (brown) and $\mathrm{Sb}$ (012) diffraction peaks. The crystallization of GeTe activates the crystallization of $\mathrm{GaSb}$, while $\mathrm{Sb}$ starts to crystallize at $210^{\circ} \mathrm{C}$ leading to a decrease in the GaSb (111) peak. 
Also, the stacked films were annealed ex-situ for $2 \mathrm{~h}$, at different temperatures, below and above the crystallization temperature of each single film, selected based on the transition temperatures determined from the R-T measurements on single films. Some differences between the in-situ and the ex-situ annealed samples can be seen in Fig. 9. One should take into account the difference in annealing times. The insitu scans were performed at a rate of $10^{\circ} \mathrm{C} / \mathrm{min}$, while the ex-situ scans at $4^{\circ} \mathrm{C} / \mathrm{min}$. The intensity of the diffraction peaks was normalized to 1 using the GaSb (111) peak. A small displacement of GaSb diffraction peaks is present, which is due to the thickness decrease and mass density change during phase transition. It was also observed an elemental segregation and a separate crystallization of the $\mathrm{Sb}$ phase after the amorphous to crystalline phase transition in $\mathrm{GaSb}$ thin films. The change in lattice constants leads to a change in film density. GaSb has a cubic structure so the displacement is equal for all orientations, but in the case of GeTe some peaks translate more than others due to the rhombohedral lattice. A small quantity of metallic Te can be observed in the ex-situ annealed samples, which is caused by the long annealing times. XRF measurements performed after annealing showed that the samples composition does not change during annealing.

EXAFS measurements performed on the ex-situ annealed samples are shown in Figs. 10 and 11. A clear change in the local environment of Ge atoms, in samples annealed at $160^{\circ} \mathrm{C}$ and $250^{\circ} \mathrm{C}$ compared to as-deposited and $100^{\circ} \mathrm{C}$ annealed films, is due to GeTe crystallization. The white lines increase in intensity and the oscillations are different in shape due to large structural changes between the amorphous and crystalline phases. The change in bonding between the crystalline and the amorphous phase is accompanied by a strong rearrangement of nearest neighbors leading to $\mathrm{Ge}-\mathrm{Ge}$ bonds formation and $\mathrm{Ge}$ coordination decrease

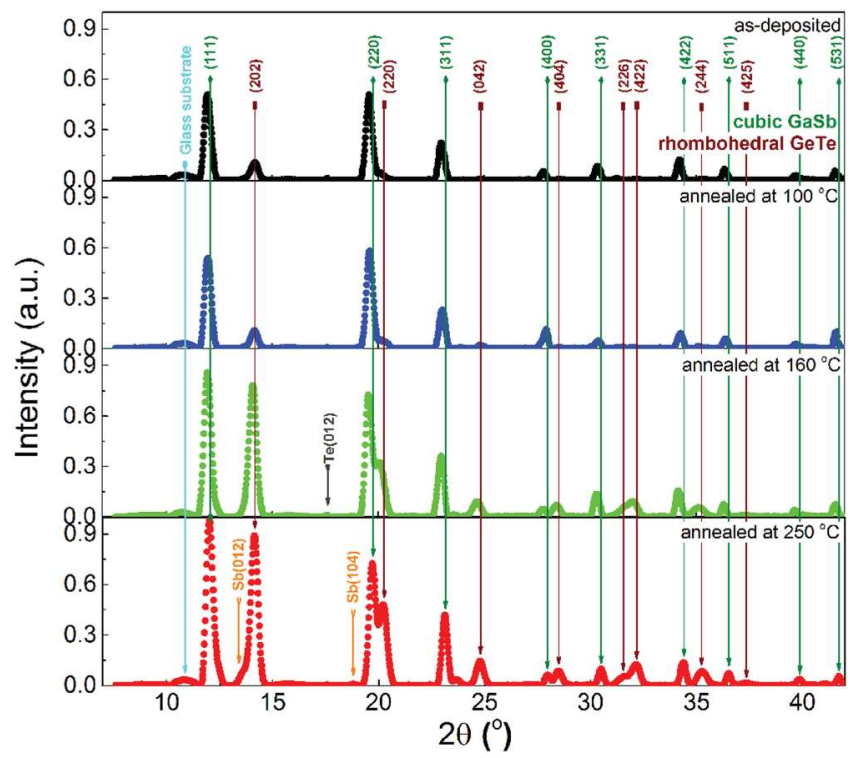

FIG. 9. XRD spectra of GeTe\GaSb stacked films, before and after ex-situ annealing at $100^{\circ} \mathrm{C}, 160^{\circ} \mathrm{C}$, and $250^{\circ} \mathrm{C}$. The crystallization of GeTe occurs in the sample annealed at $160^{\circ} \mathrm{C}$ causing a rapid growth of $\mathrm{GaSb}$ (111) peak.

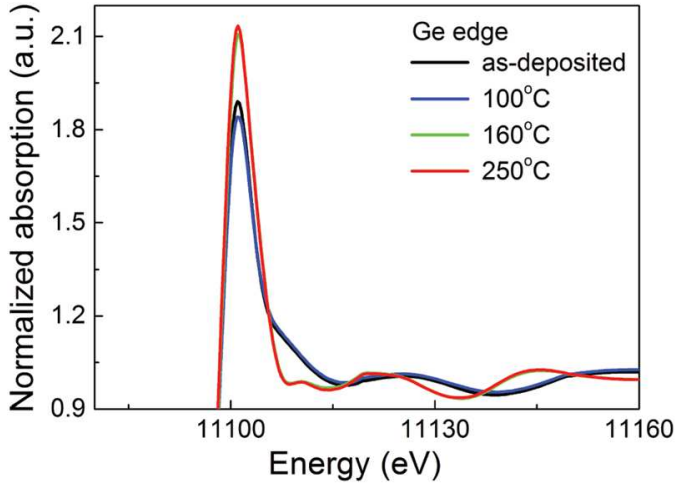

FIG. 10. XANES spectra at Ge edge of GeTelGaSb films, ex-situ annealed at $100^{\circ} \mathrm{C}, 160^{\circ} \mathrm{C}$, and $250^{\circ} \mathrm{C}$. A major change in structure is observed after GeTe crystallization.

from six to four. In order to test these hypotheses, simulations were performed and are shown in Fig. 11. For the tetrahedral configuration, only single scattering paths were considered by introducing two $\mathrm{Ge}-\mathrm{Ge}$ homopolar bonds with a distance of $2.46 \AA$ and two Ge-Te heteropolar bonds at $2.60 \AA$. For the octahedral configuration, we used single scattering paths given by three Ge-Te bonds at $2.80 \AA$ and another three $\mathrm{Ge}-\mathrm{Te}$ bonds at $3.15 \AA^{64}{ }^{64}$ The presence of homopolar Te-Te bonds was reported earlier by EXAFS analysis on GeTe crystalized thin films,${ }^{60}$ but they were not considered in our analysis. An amplitude reduction factor of 0.76 was kept fixed for all simulations at all temperatures. The good agreement between measured data and simulations in the amorphous phase suggests that Ge is four fold coordinated. The agreement is not as good for crystallized films and simulations cannot reproduce the existence of an additional feature visible between 3 and $6 \AA^{-1}$. This could be due to multiple scattering effects which were not considered in our simulations. However, the oscillations amplitude at high $\mathrm{k}$ values is well reproduced. Similar results were obtained for single films (data not shown).

The following mechanism has been proposed for phase transition: ${ }^{52}$ longer and weaker Ge-Te bonds from the crystalline structure are broken and structural segments with shorter and stronger bonds are formed, thus Ge atoms transition from the less favorable octahedral coordination to the

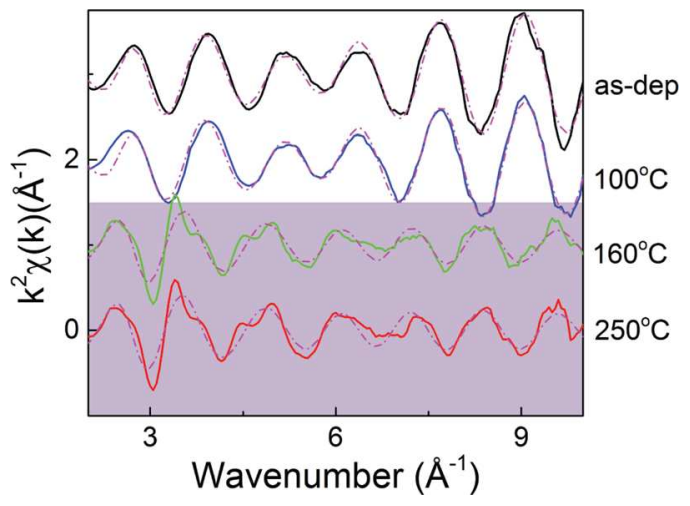

FIG. 11. $\mathrm{k}^{2}$-weighted back-Fourier transformed EXAFS oscillations at Ge edge (solid lines) and GeTe structure simulations (dashed-dotted lines, see text for explanations). The crystalline GeTe structure is highlighted. 


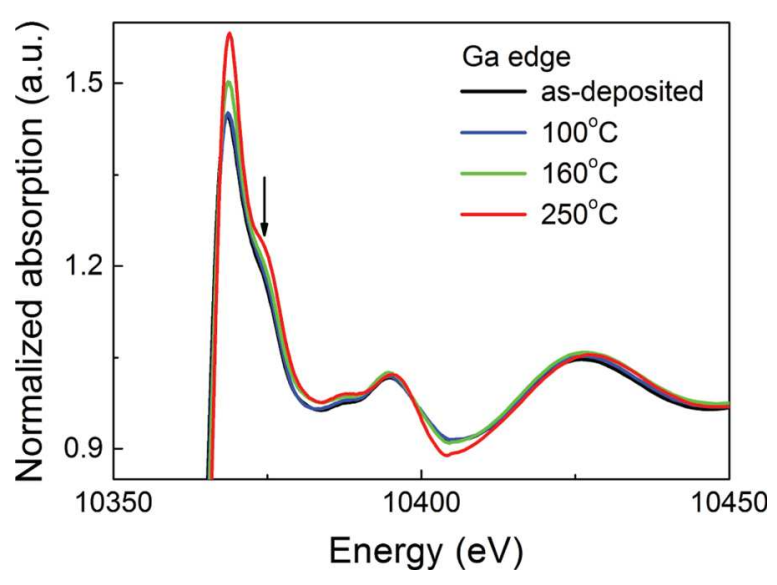

FIG. 12. XANES spectra at Ga edge of GeTelGaSb films, ex-situ annealed at $100^{\circ} \mathrm{C}, 160^{\circ} \mathrm{C}$, and $250^{\circ} \mathrm{C}$. Changes in the white line intensity are observed in the samples annealed at $160^{\circ} \mathrm{C}$ and $250{ }^{\circ} \mathrm{C}$.

tetrahedral one. $10 \%$ of Ge vacancies are present in the Ge sublattice which is crucial for reversible phase change materials. ${ }^{37}$ Such Ge vacancies and chalcogen lone pair electrons support this transformation. It is supposed that even in stoichiometric GeTe a quasi-amorphous germanium matrix is present in the crystalline state, ${ }^{47}$ which gives rise to lattice distortions. ${ }^{65}$

The structure in the local environment of $\mathrm{Ga}$, shown in Fig. 12, is more or less the same in all annealed samples, because of the small difference in structure between the crystalline and amorphous states ${ }^{56}$ and also due to the fact that the ratio of crystalline material in the as-deposited state compared to the sample annealed at $250{ }^{\circ} \mathrm{C}$ is $52 \%$. The amount of crystalline material was computed by dividing the areas of the GaSb (111) diffraction peaks in the as-deposited sample and after annealing at $250{ }^{\circ} \mathrm{C}$ (Fig. 9). Small increases in the white line intensity correlated with a shoulder around $10.375 \mathrm{keV}$ (denoted by an arrow) are signs of crystallization. The simulations shown in Fig. 13 were performed taking into account only the single scattering path given by $4 \mathrm{Sb}$ atoms present in the first shell at a distance of $2.64 \AA^{56}$ An amplitude reduction factor of 0.7 was fixed for all four simulations. The good agreement between simulations and data suggests that $\mathrm{Ga}$ is four fold coordinated in both as-deposited

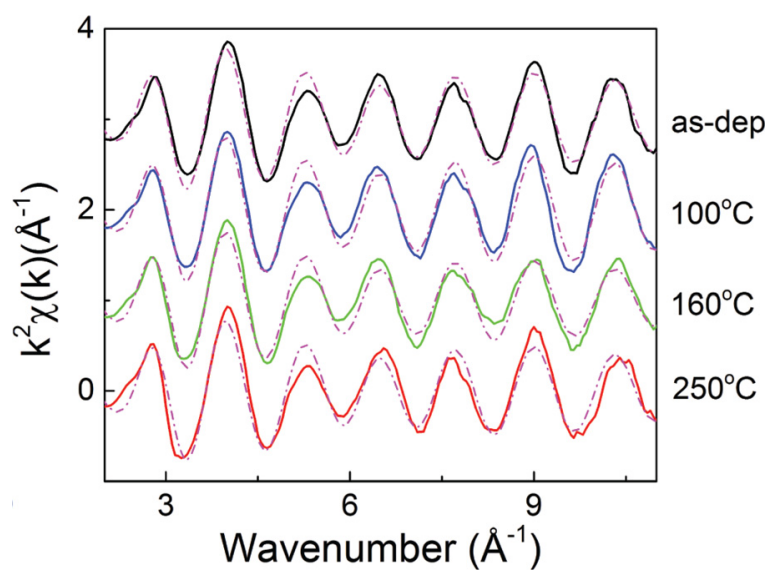

FIG. 13. $\mathrm{k}^{2}$-weighted back-Fourier transformed EXAFS oscillations at Ga edge (solid lines) and GaSb structure simulations (dashed-dotted lines). and annealed samples. Identical results were obtained for the single films (data not shown).

\section{SUMMARY}

Crystallization of germanium telluride and gallium antimony single and stacked films, prepared using pulsed laser deposition, was successfully achieved. GeTe single films were amorphous in the as-deposited state and they crystalized after reaching the transition temperature, while $\mathrm{GaSb}$ films were partially crystalline in the as-deposited state mainly due to the unavoidable formation of PLD droplets, which does not affect the crystallization temperature of the single films. The samples were slightly non-stoichiometric, which actually helped $\mathrm{GaSb}$ to increase its electrical contrast up to four orders of magnitude. A small phase separation of $\mathrm{Sb}$ was observed in the as-deposited $\mathrm{GaSb}$ films due to $\mathrm{Sb}$ excess. Ge and Te segregations were found in the ex-situ annealed GeTe samples because of the long annealing times. The stacked films showed different characteristics than single films in terms of crystallization temperatures. After GeTe crystallization, $\mathrm{Ga}$ atoms diffused from the GaSb layer into the voids of the crystalline GeTe layer. This diffusion will lead to a remaining Sb-rich GaSb layer which has a lower crystallization temperature than its single film counterpart. At higher annealing temperatures, the antimony excess from the remaining Sb-rich GaSb layer crystallizes. The crystalline $\mathrm{Sb}$ phase increases on the expense of $\mathrm{GaSb}$ crystalline phase which is reduced. This segregation is unfavorable because it is irreversible and it will deteriorate the stability and reliability performance of PCM cells. EXAFS measurements and simulations revealed that Ge is four fold coordinated in the amorphous GeTe while in the crystalline state Ge atoms are in an octahedral configuration. Ga is four fold coordinated and the structure of GaSb is similar in both asdeposited and annealed samples. In their current form, our stacked phase change materials structures are not favorable to multistate storage. With further improvements in controlling the crystallization of individual layers, the stacked structures could achieve better characteristics than their single films counterparts. Further investigations in which a buffer conductive layer is inserted between the stacked films are envisaged.

\section{ACKNOWLEDGMENTS}

The authors are grateful to Scientific Exchange NMS$\mathrm{CH}$ program for funding this study. The experiments were performed at the microXAS beamline at the Swiss Light Source, Paul Scherrer Institut, Villigen, Switzerland.

\footnotetext{
${ }^{1}$ See http://www.itrs.net for International Technology Roadmap for Semiconductors, Emerging Research Devices (2012).

${ }^{2}$ G. I. Meijer, Science 319(5870), 1625 (2008).

${ }^{3}$ M. Wuttig and N. Yamada, Nature Mater. 6, 824 (2007).

${ }^{4}$ J. Kalb, M. Wuttig, and F. Spaepen, J. Mater. Res. 22, 748 (2007).

${ }^{5}$ D. Lencer, M. Salinga, and M. Wuttig, Adv. Mater. 23, 2030 (2011).

${ }^{6}$ J. Hegedus and S. R. Elliot, Nature Mater. 7, 399 (2008).

${ }^{7}$ M. Popescu, F. Sava, A. Velea, and A. Lörinczi, J. Non-Cryst. Solids 355, $1820(2009)$

${ }^{8}$ A. Velea, J. Non-Cryst. Solids 357, 2626 (2011).
} 
${ }^{9}$ D. Loke, T. H. Lee, W. J. Wang, L. P. Shi, R. Zhao, Y. C. Yeo, T. C. Chong, and S. R. Elliott, Science 336, 1566 (2012).

${ }^{10}$ G. Bruns, P. Merkelbach, C. Schlockermann, M. Salinga, M. Wuttig, T. D. Happ, J. B. Philipp, and M. Kund, Appl. Phys. Lett. 95, 043108 (2009).

${ }^{11}$ S. Raoux, A. K. Konig, H.-Y. Cheng, D. Garbin, R. W. Cheek, J. L. Jordan-Sweet, and M. Wuttig, Phys. Status Solidi B 249, 1999 (2012).

${ }^{12}$ K. L. Chopra and S. K. Bahl, J. Appl. Phys. 40, 4171 (1969).

${ }^{13}$ M. K. Santala, B. W. Reed, S. Raoux, T. Topuria, T. LaGrange, and G. H. Campbell, Appl. Phys. Lett. 102, 174105 (2013).

${ }^{14}$ D. Lencer, M. Salinga, B. Grabowski, T. Hickel, J. Neugebauer, and M. Wuttig, Nature Mater. 7, 972 (2008).

${ }^{15}$ T.-Y. Lee, K.-B. Kim, B.-K. Cheong, T. S. Lee, S. J. Park, K. S. Lee, W. M. Kim, and S. G. Kim, Appl. Phys. Lett. 80, 3313 (2002).

${ }^{16}$ D. J. Gravesteijn, Appl. Opt. 27, 736 (1988).

${ }^{17}$ T. Matsunaga, J. Akola, S. Kohara, T. Honma, K. Kobayashi, E. Ikenaga, R. O. Jones, N. Yamada, M. Takata, and R. Kojima, Nature Mater. 10, 129 (2011).

${ }^{18}$ Y.-F. Lai, J. Feng, B.-W. Qiao, X.-G. Huang, Y.-F. Cai, Y.-Y. Lin, and T.A. Tang, Chin. Phys. Lett. 23, 2516 (2006).

${ }^{19}$ Y. Hu, M. Sun, S. Song, Z. Somg, and J. Zhai, Integrated Ferroelectr. 140, 8 (2012).

${ }^{20}$ X. Zhou, L. Wu, Z. Song, F. Rao, K. Ren, C. Peng, S. Song, B. Liu, L. Xu, and S. Feng, Appl. Phys. Lett. 103, 072114 (2013).

${ }^{21}$ N. Papandreou, H. Pozidis, T. Mittelholzer, G. F. Close, M. Breitwisch, C. Lam, and E. Eleftheriou, in IEEE International Memory Workshop (IMW) (2011).

${ }^{22}$ F. Bai, H. Zhang, S. Gupta, and S. Kurinec, Mater. Sci. Forum 687, 677 (2011).

${ }^{23}$ A. Devasia, D. MacMahon, S. Raoux, K. A. Campbell, and S. K. Kurinec, Thin Solid Films 520, 3931 (2012).

${ }^{24}$ C.-F. Chen, A. Schrott, M. H. Lee, S. Raoux, Y. H. Shih, M. Breitwisch, F. H. Baumann, E. K. Lai, T. M. Shaw, P. Flaitz, R. Cheek, E. A. Joseph, S. H. Chen, B. Rajendran, H. L. Lung, and C. Lam, in IEEE International Memory Workshop (2009).

${ }^{25}$ A. Velea, M. Popescu, A. Lörinczi, F. Sava, I.-D.Simandan, and C. Mihai, J. Optoelectron. Adv. Mater. 13, 1594 (2011).

${ }^{26}$ S. Raoux, R. M. Shelby, J. Jordan-Sweet, B. Munoz, M. Salinga, Y.-C. Chen, Y.-H. Shih, E.-K. Lai, and M.-H. Lee, Microelectron. Eng. 85, 2330 (2008).

${ }^{27}$ L. van Pieterson, M. H. R. Lankhorst, M. van Schijndel, A. E. T. Kuiper, and J. H. J. Roosen, J. Appl. Phys. 97, 083520 (2005).

${ }^{28}$ M. N. Schneider, P. Urban, A. Leineweber, M. Döblinger, and O. Oeckler, Phys. Rev. B 81, 184102 (2010).

${ }^{29}$ M. Frumar, B. Frumarova, P. Nemec, T. Wagner, J. Jedelsky, and M. Hrdlicka, J. Non-Cryst. Solids 352, 544 (2006).

${ }^{30}$ V. Nazabal, M. Cathelinaud, W. Shen, P. Nemec, F. Charpentier, H. Lhermite, M. L. Anne, J. Capoulade, F. Grasset, A. Moreac, S. Inoue, M. Frumar, J. L. Adam, M. Lequime, and C. Amra, Appl. Opt. 47, C114 (2008).

${ }^{31}$ P. Němec, M. Frumar, J. Jedelský, M. Jelínek, J. Lančok, and I. Gregora, J. Non-Cryst. Solids 299-302, 1013 (2002).

${ }^{32}$ M. J. Schoning, C. Schmidt, J. Schubert, W. Zander, S. Mesters, P. Kordos, H. Lutha, A. Leginc, B. Seleznev, and Y. G. Vlasov, Sens. Actuators B 68, 254 (2000).

${ }^{33}$ K. E. Youden, T. Grevatt, R. W. Eason, H. N. Rutt, R. S. Deol, and G. Wylangowski, Appl. Phys. Lett. 63, 1601 (1993).

${ }^{34}$ M. Popescu, A. Velea, F. Sava, A. Lorinczi, A. Tomescu, C. Simion, E. Matei, G. Socol, I. N. Mihailescu, A. Andronie, and I. Stamatin, Phys. Status Solidi A 207, 516 (2010).
${ }^{35}$ E. Morales-Sanchez, E. Prokhorov, J. Gonzalez-Hernandez, and A. Mendoza-Galvan, Thin Solid Films 471, 243 (2005).

${ }^{36}$ S. Raoux, J. L. Jordan-Sweet, and A. Kellock, J. Appl. Phys. 103, 114310 (2008).

${ }^{37}$ M. Chen, K. A. Rubin, and R. W. Barton, Appl. Phys. Lett. 49, 502 (1986).

${ }^{38}$ S. Raoux, H.-Y. Cheng, M. A. Caldwell, and H.-S. P. Wong, Appl. Phys. Lett. 95, 071910 (2009).

${ }^{39}$ I. Friedrich, V. Weidenhof, W. Njoroge, P. Franz, and M. Wuttig, J. Appl. Phys. 87, 4130 (2000).

${ }^{40}$ C. N. Borca, D. Grolimund, M. Willimann, B. Meyer, K. Jefimovs, J. VilaComamala, and C. David, J. Phys.: Conf. Ser. 186, 012003 (2009).

${ }^{41}$ G. K. Williamson and W. H. Hall, Acta. Metal. 1, 22 (1953).

${ }^{42}$ See http://xrdua.ua.ac.be/ for XRDUA, 2D Powder-XRD Analysis.

${ }^{43}$ See http://www.crystalimpact.com/match/ for Match! Phase Identification from Powder Diffraction.

${ }^{44}$ B. Ravel and M. Newville, J. Synchrotron Radiat. 12, 537 (2005).

${ }^{45}$ Y. Maeda and M. Wakagi, Jpn. J. Appl. Phys., Part 1 30, 101 (1991).

${ }^{46}$ R. Mazelsky, M. S. Lubell, and W. E. Kramer, J. Chem. Phys. 37, 45 (1962).

${ }^{47}$ A. V. Kolobov, J. Tominaga, P. Fons, and T. Uruga, Appl. Phys. Lett. 82, 382-384 (2003).

${ }^{48}$ O. Uemura, Y. Sagara, M. Tsushima, T. Kamikawa, and T. Satow, J. NonCryst. Solids 33, 71 (1979).

${ }^{49}$ G. B. Fisher, J. Tauc, and Y. Verhelle, in Amorphous and Liquid Semiconductors, edited by J. Stuke (Taylor and Francis, London, 1974), p. 1259.

${ }^{50}$ S. J. Pickart, Y. P. Sharma, and J. P. de Neufville, J. Non-Cryst. Solids 34, 183 (1979).

${ }^{51}$ P. Boolchand, B. B. Triplett, S. S. Hanna, and J. P. de Neufville, Mossbauer Effect Methodology (Plenum, New York, 1974).

${ }^{52}$ A. V. Kolobov, P. Fons, J. Tominaga, A. L. Ankudinov, S. N. Yannopoulos, and K. S. Andrikopoulos, J. Phys.: Condens. Matter 16, S5103 (2004).

${ }^{53}$ D. Ielmini and Y. Zhang, Appl. Phys. Lett. 90, 192102 (2007).

${ }^{54}$ S. K. Bahl and K. L. Chopra, J. Appl. Phys. 41, 2196 (1970).

${ }^{55}$ S. Raoux, H.-Y. Cheng, B. Muñoz, and J. Jordan-Sweet, in Proceedings of the 8th Europ. Phase Change and Ovonic Sci. Symp. E\PCOS (2009).

${ }^{56}$ A. V. Sapelkin, S. C. Bayliss, A. G. Lyapin, V. V. Brazhkin, and A. J. Dent, Phys. Rev. B 56, 11531 (1997).

${ }^{57}$ H. Y. Cheng, S. Raoux, and J. L. Jordan-Sweet, in Proceedings of the 10th Europ. Phase Change and Ovonic Sci. Symp. E\PCOS (2011).

${ }^{58}$ S. Raoux, C. Cabral, L. Krusin-Elbaum, J. L. Jordan-Sweet, K. Virwani, M. Hitzbleck, M. Salinga, A. Madan, and T. L. Pinto, J. Appl. Phys. 105, 064918 (2009).

${ }^{59}$ J. I. Langford, R. J. Cernik, and O. Louer, J. Appl. Cryst. 24, 913 (1991).

${ }^{60}$ M. Putero, M.-V. Coulet, T. Ouled-Khachroum, C. Muller, C. Baehtz, and S. Raoux, Appl. Phys. Lett. 103, 231912 (2013).

${ }^{61}$ N. Kaiser, Thin Solid Films 116, 259 (1984).

${ }^{62}$ M. Kaiser, L. van Pieterson, and M. A. Verheijen, J. Appl. Phys. 96, 3193 (2004).

${ }^{63}$ J. M. Del Pozo, M. P. Herrero, and L. Diaz, J. Non-Cryst. Solids 185, 183 (1995).

${ }^{64}$ Y. G. Choi, A. Kovalskiy, B.-K. Cheong, and H. Jain, Chem. Phys. Lett. 534, 58 (2012).

${ }^{65}$ T. Matsunaga, H. Morita, R. Kojima, N. Yamada, K. Kifune, Y. Kubota, Y. Tabata, J.-J. Kim, M. Kobata, E. Ikenaga, and K. Kobayashi, J. Appl. Phys. 103, 093511 (2008). 